\title{
LA-UR-15-21390
}

Approved for public release; distribution is unlimited.

Title: $\quad$ SESAME Equations of State for Stress Cushion and Related Materials

Author(s): $\quad$ Coe, Joshua Damon

Intended for: Report

Issued: 
Disclaimer:

Los Alamos National Laboratory, an affirmative action/equal opportunity employer,is operated by the Los Alamos National Security, LLC for the National NuclearSecurity Administration of the U.S. Department of Energy under contract DE-AC52-06NA25396. By approving this article, the publisher recognizes that the U.S. Government retains nonexclusive, royalty-free license to publish or reproduce the published form of this contribution, or to allow others to do so, for U.S. Government purposes. Los Alamos National Laboratory requests that the publisher identify this article as work performed under the auspices of the U.S. Departmentof Energy. Los Alamos National Laboratory strongly supports academic freedom and a researcher's right to publish; as an institution, however, the Laboratory does not endorse the viewpoint of a publication or guarantee its technical correctness. 


\section{SESAME Equations of State for Stress Cushion and Related Materials}

Joshua D. Coe ${ }^{a}$

Theoretical Division, Los Alamos National Laboratory, Los Alamos, NM, 87545

(Dated: 12 February 2015)

I examine LANL equations of state (EOS) for stress cushion and related materials, namely S5370, SX358, and Sylgard 184. In the the first two cases, the SESAME library contains entries for both the inert (unreacted) and decomposition products. I compare inert EOS results with ambient property measurements to the extent possible, then I check the compositions used to build the products tables. I plot the predicted Hugoniots alongside the available shock data, then draw some conclusions.

a)Electronic mail: jcoe@lanl.gov 


\section{INTRODUCTION}

Here I review the status of LANL equations of state (EOS) for stress cushion and related materials. These comprise S5370, its replacement (SX358), and Dow Corning's Sylgard $184^{\mathrm{TM}}$ (hereafter, Sylgard). Together these are represented by 6 different SESAME tables in the current library. In Table I I list their numbers and pedigree, taken directly from the 101 subtables. Both S5370 and SX358 are assigned separate entries for inert and decomposition products.

This report is structured in three parts. First I compare ambient properties read from the inert tables with the little data I could find, as well as with one another. Given the close relationship between the three materials, the naive expectation is that property values should be quite similar. Next I check key inputs of the products modeling: chemical composition (in the form of elemental abundance ratios), and heat of formation. I took some pains to verify that the composition underlying the products tables corresponded well with the best sources I could find. Finally, I examine the predicted shock response of both full-density and porous material.

I have several points of interest. First, to analyze the fidelity of these EOS to the available data. Secondly, to compare them with one another so as to assess the extent to which their use might be interchangeable. This is more than a mere convenience: there is little data on any of these materials, but by far the most on Sylgard. The extent to which Sylgard is not an adequate proxy for S5370/SX358 is the extent to which more data on the latter might be helpful. If possible, I am also interested in identifying when these materals decompose under shock loading, and the degree of volume collapse upon reaction.

\section{INERT PROPERTIES}

The inert tables all were based on the following decomposition of the Helmholtz free energy $^{1-3}$

$$
F(\rho, T)=\phi(\rho)+F_{\text {ion }}(\rho, T)+F_{\text {elec }}(\rho, T) .
$$

The first term on the rhs is the energy of the static lattice at $0 \mathrm{~K}$, where all ions and electrons are in their ground state. This "cold curve" is not equivalent to the zero temperature isotherm, as it does not include zero point energy (zpe). The second term is the free energy 
TABLE I. Summary of existing SESAME tables for stress cushion and related materials.

\begin{tabular}{lcc}
\hline \hline Material & Library Number & Author (Year) \\
\hline Sylgard 184 & 7930 & F. Dowell (1984), J. C. Boettger \& S. Crockett (2001) \\
Sylgard 184 & 7931 & F. Dowell (1985), J. C. Boettger \& S. Crockett (2001) \\
S5370 (inert) & 7970 & S. Crockett (2007) \\
S5370 (products) & 7971 & M. Sam Shaw \& S. Crockett (2007) \\
SX358 (inert) & 7980 & S. Crockett (2007) \\
SX358 (products) & 7981 & M. Sam Shaw \& S. Crockett (2007) \\
\hline \hline
\end{tabular}

of ionic excitations with electrons in their ground state, and the last is the free energy of electronic excitations with the ions in their ground state. In order for the model to be complete, there should be an additional term coupling the ions and electrons; this coupling is generally assumed to be small, and therefore is often neglected. All other thermodynamic potentials may be obtained by Legendre transformation of (1), and various observables found by application of appropriate derivatives. For example, the internal energy is

$$
E=F-T\left(\frac{\partial F}{\partial T}\right)_{\rho},
$$

and the pressure is

$$
P=\rho^{2}\left(\frac{\partial F}{\partial \rho}\right)_{T} .
$$

Equation (1) thus constitutes as complete a description of a material as classical thermodynamics can provide. We express otherwise extensive quantities in specific units throughout.

Inert EOS for all three materials were based on roughly the same procedure. ${ }^{4}$ The cold curve was obtained by a linear or quadratic fit to Hugoniot data in the $U_{s}-u_{p}$ plane. A Debye-like model $^{5}$ was used for the ionic contribution, and Thomas-Fermi-Dirac ${ }^{6,7}$ theory for the thermal electronic.

The Sylgard EOS contained some additional wrinkles that I had not encountered previously: decomposition of the characteristic temperature into inter- and intrachain components $^{8}$ and variation of this parameter at a threshold compression of $1.42\left(u_{p} \approx 1\right)$. The threshold is meant to signal a conversion of the repeat unit from $\mathrm{Si}\left(\mathrm{CH}_{3}\right)_{2} \mathrm{O}$ to $\mathrm{Si}\left(\mathrm{CH}_{3}\right)_{2} \mathrm{O}$. Thus, the model attempts to capture chemistry (in this case, a cross-linking phase transformation) through abrupt variation of the characteristic temperature. The cut- 
off appears to have been a function of compression only, independent of temperature. Aside from making no sense physically, I would expect such a discontinuity to cause problems in hydrodynamic simulation. This feature appears to be have been removed in 7931, and may be the reason that the 102 subtable "strongly recommends" its use over that of 7930 .

Table II shows results of interpolating the inert tables to the ambient state $(P=1$ bar, $T=298 \mathrm{~K})$. Experimental data, where available, appear in parenthesis beneath a calculated entry. Properties examined include the density $\left(\rho_{0}\right)$, isothermal bulk modulus $\left(B_{T}\right)$, isentropic bulk modulus $\left(B_{s}\right)$, isobaric specific heat $\left(C_{p}\right)$, coefficient of thermal expansion (CTE, $\alpha$ ) and Grüneisen gamma. I found almost no such data for SX358 or S5370, and even that for Sylgard requires some qualification. The isentropic bulk modulus listed is simply the extrapolation to zero particle velocity of a quadratic fit to the Hugoniot. All of the isothermal values (based on diamond anvil cell experiments) reported in Ref. 9 are higher than this, which would violate thermodynamic stability. Thermodynamic consistency demands that

$$
B_{s}=\frac{B_{T}}{1-\alpha T \Gamma}
$$

where

$$
\Gamma=\frac{\alpha B_{T}}{\rho C_{p}} .
$$

If one simply accepts the extrapolated value for $B_{s}$, then $B_{T} \approx 1.225$ and $\Gamma \approx 0.89$. SESAMEs 7930/1 perform an adequate job (at best) of replicating these results. The $\Gamma$ from 7930 seems quite low. Justification for the differences between 7970 and 7980 is unclear to me. The $\Gamma$ values are very similar, but only because the moduli and CTE differ by roughly equal factors in the opposite direction (cf. equation (5)). The $\Gamma$ value itself was set to match porous shock data (not shown). ${ }^{4}$ All of this underlines an important concept well-known in the EOS world: shock data by themselves are a weak constraint. Because shock pressures arise from a combination of thermal and mechanical response (see equation (1)), it is almost always straightforward to balance those contributions so as to recover the sum desired.

\section{COMPOSITION}

The products EOS used to build 7971 and 7981 were calculated as an ideal mixture ${ }^{15}$ of fluids and bulk carbon in full chemical equilibrium. This methodology is typically applied 
TABLE II. Ambient properties as given by the SESAME EOS tables. Experimental values are provided in parenthesis below the EOS result.

\begin{tabular}{ccccccc}
\hline \hline Table & $\rho_{0}(\mathrm{~g} / \mathrm{cc})$ & $B_{T}(\mathrm{GPa})$ & $B_{S}(\mathrm{GPa})$ & $C_{p}(\mathrm{MJ} / \mathrm{kg} \mathrm{K})$ & $\alpha(1 / \mathrm{K})$ & $\Gamma$ \\
\hline 7930 & 1.047 & 1.257 & 1.294 & $2.841 \mathrm{E}-3$ & $4.759 \mathrm{E}-4$ & 0.207 \\
7931 & 1.038 & 2.634 & 3.689 & $3.683 \mathrm{E}-3$ & $1.179 \mathrm{E}-3$ & 1.138 \\
& $(1.037)$ & $1.225^{10}$ & $(1.54)^{11}$ & $(1.6 \mathrm{E}-3)^{12}$ & $(9.6 \mathrm{E}-4)^{13}$ & $(0.89)^{10}$ \\
7970 & 1.014 & 2.250 & 2.297 & $2.215 \mathrm{E}-3$ & $2.628 \mathrm{E}-4$ & 0.269 \\
& $(1.014)$ & & & & & \\
7980 & 1.142 & 4.385 & 4.449 & $2.188 \mathrm{E}-3$ & & \\
& $(1.117)^{14}$ & & & & & \\
\hline \hline
\end{tabular}

only over a domain of $\mathrm{T} \approx 1000-10000 \mathrm{~K}$ in temperature and $\eta \approx 10^{-4}-2$ in compression. The resultant "core" table was then extended to cover the standard SESAME domain of $T=0-10 \mathrm{keV}$ and $\eta=0-20000$, based on methodology described in Ref. 16.

Fluid EOS were built using Ross perturbation theory, ${ }^{17}$ and carbon (in the form of diamond) was treated with equation (1) sans the thermal electronic contribution. ${ }^{\text {a }}$ Because all constituent models were calibrated to data for the components in isolation, the primary "knobs" for the mixture EOS are its chemical composition and the heat of formation (HOF) of the starting material. For chemically complex composites such as those under survey, precise data on either of those features may be lacking. To the extent that one is known, the other can be tuned to match data. A well-established composition is, in other words, a strong constraint.

My principal source for the composition of SX358 and S5370 was a 2006 tech report. ${ }^{18}$ As is often the case, compositions were given as wt\% of molecular and polymeric precursors, whereas our chemical equilibrium models require elemental abundance ratios. Because the translation from one to the other is a tedious and error-prone exercise, I catalogued the procedure in some detail in Tables III and IV. For the four leftmost columns I relied entirely on Ref. 18 and standard atomic weights. In the case of polymeric components, I approximated the number of monomers in a "molecule" from the formula of the repeating

\footnotetext{
${ }^{a}$ Diamond is an excellent insulator over the relevant domain.
} 
TABLE III. Compositional details of SX358, as derived from LA-UR-06-0740. I calculated the number of repeating units in a polymer by dividing the MW of the monomer into that given for the constituent as a whole. Final compositions on a per mol basis are given in Table V.

\begin{tabular}{|c|c|c|c|c|c|c|}
\hline name & formula & $\mathrm{wt} \%$ & MW & $\overline{\mathrm{n}}$ & $\frac{\mathrm{mol}}{1 \mathrm{~kg} \mathrm{SX} 358}$ & mol frac \\
\hline PDMS & {$\left[\mathrm{C}_{2} \mathrm{H}_{6} \mathrm{OSi}\right]_{n}$} & 17.19 & 2350 & 31.69 & $7.315 \mathrm{E}-2$ & $2.527 \mathrm{E}-2$ \\
\hline PDMS & {$\left[\mathrm{C}_{2} \mathrm{H}_{6} \mathrm{OSi}\right]_{n}$} & 14.45 & 29400 & 396.5 & $4.915 \mathrm{E}-3$ & $1.698 \mathrm{E}-3$ \\
\hline PDMS & {$\left[\mathrm{C}_{2} \mathrm{H}_{6} \mathrm{OSi}\right]_{n}$} & 43.36 & 54300 & 732.3 & $7.985 \mathrm{E}-3$ & $2.759 \mathrm{E}-3$ \\
\hline diphenylmethylsilanol & $\mathrm{C}_{13} \mathrm{H}_{14} \mathrm{OSi}$ & 5.00 & 214.339 & - & $2.333 \mathrm{E}-1$ & $8.062 \mathrm{E}-2$ \\
\hline PMHS & {$\left[\mathrm{CH}_{3} \mathrm{HSiO}\right]_{n}$} & 3.00 & 12150 & 202.1 & $2.469 \mathrm{E}-3$ & $8.531 \mathrm{E}-4$ \\
\hline tetrapropylorthosilicate & $\left(\mathrm{CH}_{3} \mathrm{CH}_{2} \mathrm{CH}_{2} \mathrm{O}\right)_{4} \mathrm{Si}$ & 2.00 & 264.437 & - & $7.563 \mathrm{E}-2$ & $2.613 \mathrm{E}-2$ \\
\hline diatomaceous earth & $\mathrm{SiO}_{2}$ & 15.00 & 60.083 & - & 2.497 & 0.8628 \\
\hline
\end{tabular}

unit and the molecular weights (MW) listed in Table 2 of Ref. 18. Given the wt\% and MW of each constituent, I found the mass present in $1 \mathrm{~kg}$ of material; from this, I obtained the mol fractions. Elemental abundances were then calculated from a mol-fraction-weighted sum of the constituent formulas, normalized to hydrogen.

The one complicating factor in this procedure was that viscosities, rather than MWs, were provided for the polymeric precursors of S5370. For the higher viscosity PDMS I used an empirical relation derived specifically for that material ${ }^{19}$

$$
\log \eta_{0}=1.00+0.0123 \sqrt{\mathrm{MW}}
$$

where $\eta_{0}$ is the viscosity (in centistokes) at $25^{\circ} \mathrm{C}$. This expression was not intended for viscosities less than 2500 cs, so for low-viscosity PDMS and PMHS I used a linear fit to MW-viscosity data (PDMS only) I obtained directly from Dow Corning. ${ }^{20}$. Elemental ratios for Sylgard were given explicitly in Ref. 11, based on results reported in Ref. 12. I have not been able to locate the latter, so I'm unsure by what method they were obtained.

Mine is an inexact procedure for calculating composition. Polymeric contributions were restricted to the repeating unit, neglecting end groups. Based on the approximate length of these chains, I suspect that the correction is negligible. I've also ignored cross-linking reactions, which often produce volatile components (such as $\mathrm{H}_{2}$, see Scheme 1 in Ref. 18) 
TABLE IV. Compositional details of S5370, as derived from LA-UR-06-0740. I calculated the number of repeating units in a polymer by dividing the MW of the monomer into that given for the constituent as a whole. Final compositions on a per mol basis are given in Table V.

\begin{tabular}{lcccccc}
\hline \hline name & formula & wt\% & MW & $\overline{\mathrm{n}}$ & $\frac{m}{1 \mathrm{~kg} \mathrm{SX} 358}$ & mol frac \\
\hline PDMS & {$\left[\mathrm{C}_{2} \mathrm{H}_{6} \mathrm{OSi}\right]_{n}$} & 12.0 & 2633 & 35.51 & $4.558 \mathrm{E}-2$ & $1.573 \mathrm{E}-2$ \\
PDMS & {$\left[\mathrm{C}_{2} \mathrm{H}_{6} \mathrm{OSi}\right]_{n}$} & 60.0 & 35000 & 472.0 & $1.714 \mathrm{E}-2$ & $5.914 \mathrm{E}-3$ \\
diphenylmethylsilanol & $\mathrm{C}_{13} \mathrm{H}_{14} \mathrm{OSi}$ & 5.00 & 214.339 & - & $2.333 \mathrm{E}-1$ & $8.050 \mathrm{E}-2$ \\
PMHS & {$\left[\mathrm{CH}_{3} \mathrm{HSiO}\right]_{n}$} & 6.00 & 2067 & 34.38 & $2.903 \mathrm{E}-2$ & $1.002 \mathrm{E}-2$ \\
tetrapropylorthosilicate & $\left(\mathrm{CH}_{3} \mathrm{CH}_{2} \mathrm{CH}_{2} \mathrm{O}\right)_{4} \mathrm{Si}$ & 2.00 & 264.437 & - & $7.563 \mathrm{E}-2$ & $2.610 \mathrm{E}-2$ \\
diatomaceous earth & $\mathrm{SiO}_{2}$ & 15.00 & 60.083 & - & 2.497 & 0.8616 \\
\hline \hline
\end{tabular}

that would then off-gas. Again, I expect the correction to be small. Finally, I estimated the MW of PMHS in S5370 based on interpolation of PDMS data. I have less sense for the magnitude of this error, but given the small difference in structure (a methyl replaced by a hydrogen on the siloxy group) and the low wt\% of PMHS, I again expect it to be small. Regardless, the formulations I obtained for SX358 and S5370 agreed with those used to build 7971 and 7981 to three or more significant digits. The compositions are probably as accurate as could be expected, and consequent uncertainties in the EOS should be minimal.

As seen in Table V, the composition of all three materials is quite similar, SX358 and S5370 being nearly identical. In my experience, the shape of the products locus is dictated primarily by composition, whereas its "lateral" placement in the $P-V$ plane is strongly affected by the heat of formation. The heats of formation for 7971 and 7981 were both $-168.7 \mathrm{kcal} / \mathrm{mol}$. This is a fairly large number in my opinion, but given the high degree of confidence in the compositions I suspect it was simply tuned to match data.

\section{SHOCK RESPONSE}

The primary source of EOS data on cushion materials is shock wave experiments, all performed here at LANL. The LASL Compendium (a.k.a. Marsh) ${ }^{21}$ contains an extensive set for Sylgard. Joe Fritz collected data on S5370 that were, to my knowledge, never 
published. More recently, Dattelbaum performed a series of gas gun shots on S5370 and SX358 over a range of initial densities. ${ }^{14}$

\section{A. Full-Density Material}

Figures 1 and 2 are of the data only. The most extensive set by far is for Sylgard, followed by that of Fritz. Sylgard is slightly stiffer than the other two materials, which themselves respond quite similarly. Any volume collapse upon decomposition is small in every case. In Figures 3-8 I plot the predicted Hugoniots alongside the data. Due to the qualitative nature of this analysis, I did not attempt to refine the resolution of the grids in order to obtain smooth curves; my conclusions were unaffected.

Figure 3 shows clearly that the compression threshold was not used in 7931, which also does a better job than 7930 of recovering the high-pressure limit. The discontinuity in 7930 is not one we typically assign to full decomposition, as this typically occurs only for stronger shocks $\left(u_{p} \approx 2.5-3 \mathrm{~km} / \mathrm{s}\right.$ and $\left.P \approx 20-30 \mathrm{GPa}\right) \cdot{ }^{22,23}$ Two different linear $U_{s}-u_{p}$ fits at low particle velocity have sometimes been used to replicate the concave nature of the locus in this regime; ${ }^{24}$ however, we now assign this feature to removal of "porosity" broadly construed. ${ }^{25,26}$ SESAME 7931 does a pretty good job with all the data for $u_{p}>1 \mathrm{~km} / \mathrm{s}$, such that it is very difficult to identify any discontinuity due to reaction.

The combination of 7970 and 7971 seem to indicate a decomposition threshold for S5370 of $u_{p} \approx 3.5-4 \mathrm{~km} / \mathrm{s}$ and $P \approx 25 \mathrm{GPa}$. Precise estimates are complicated by the small change in volume, as well as the scatter in the data. This threshold particle velocity is slightly higher

TABLE V. Final compositions (per mol, normalized to hydrogen) and reference densities for the materials under survey.

\begin{tabular}{lccc}
\hline \hline Material & \multicolumn{1}{c}{ Composition } & $\overline{\mathrm{A}}$ & $\overline{\mathrm{Z}}$ \\
\hline Sylgard 184 & $\mathrm{C}_{0.351} \mathrm{HO}_{0.203} \mathrm{Si}_{0.184}$ & 7.84 & 4.20 \\
S5370 (inert) & $\mathrm{C}_{0.360} \mathrm{HO}_{0.240} \mathrm{Si}_{0.200}$ & 8.21451 & 4.37696 \\
S5370 (products) & $\mathrm{C}_{0.360} \mathrm{HO}_{0.240} \mathrm{Si}_{0.200}$ & 8.21451 & 4.37696 \\
SX358 (inert) & $\mathrm{C}_{0.362} \mathrm{HO}_{0.237} \mathrm{Si}_{0.197}$ & 8.1773 & 4.35894 \\
SX358 (products) & $\mathrm{C}_{0.362} \mathrm{HO}_{0.237} \mathrm{Si}_{0.197}$ & 8.1773 & 4.35894 \\
\hline \hline
\end{tabular}


than is typical in my experience. ${ }^{25,27}$ The Dattelbaum shots are almost certainly unreactive, and lie well above the data and EOS.

With one exception, SESAME 7980 does a good job of recovering the Dattelbaum shots for SX358. In the absence of actual products data, it appears to me that 7981 was fit to capture the high-pressure limit of Sylgard. It is impossible to identify any sort of threshold, and reactant/products loci are basically indistinguishable at high pressure.

\section{B. Porous Material}

Thresholds for shock-driven decomposition drop rapidly with initial density. ${ }^{25}$ While the Dattelbaum data on full-density SX358 almost certainly do not exceed the threshold, I believe that they do in the case of porous material. Before examining the results, a word is in order about how such information was extracted from the EOS tables. For all table queries I used the OpenSesame software package. ${ }^{28}$ For the inerts, I simply requested a Hugoniot centered at ambient pressure and the porous $\rho_{0}$ of interest. This, of course, yields an $E_{0}$ higher than that at full density under the same conditions. The origin of the products locus was slightly more complicated, and in order to motivate its selection I'll refer to the jump condition

$$
E_{H}=E_{0}+\frac{1}{2}\left(P_{0}+P_{H}\right)\left(\frac{1}{\rho_{0}}-\frac{1}{\rho_{H}}\right) .
$$

When solving for the products locus, the final (Hugoniot, ' $H^{\prime}$ ) states lie on the EOS surface of the products, whereas the initial states ( $\left.{ }^{\circ}{ }^{\prime}\right)$ lie on the EOS surface of the inert. In order to pull the Hugoniot from the products table, one must enter an initial state not lying on the EOS surface. This is simple enough for the pressure (ambient) and density (the porous density of interest), but $E_{0}$ is less clear. Here I had the benefit of the input decks Sam Shaw used to create the limited-range 7981 table with the eos 2 ses code. ${ }^{29}$ The zero of energy in eos2ses is isolated elements at $0 \mathrm{~K}$ and ambient pressure, whereas many modern inert tables have been "standardized" such that the energy at STP is zero. In order to align the zeros correctly (and thereby ensure the proper energy release upon decomposition), a uniform shift is applied to the products table. In the case of 7981 , this was $7.0584 \mathrm{MJ} / \mathrm{kg}$. ${ }^{\mathrm{b}}$ This is

b See my previous comment (Section III) on this number being unusually large in the cases of SX358 and S5370. The translation from per mol to per mass units involves a factor of the molecular weight, and I am unclear as to how Sam estimated this. 
the $\mathrm{HOF}$ in specific units, plus the energy required to heat the isolated elements from 0 to $298 \mathrm{~K}$. So the products locus was centered at an $E_{0}$ of $-7.0584 \mathrm{MJ} / \mathrm{kg}$.

I show the Hugoniots given by SESAME 7980 and 7981 alongside gas gun data for $\left\langle\rho_{0}\right\rangle=0.727$ and $0.4125 \mathrm{~g} / \mathrm{cc}$. I discarded one of the $0.4125 \mathrm{~g} / \mathrm{cc}$ shots, ${ }^{\mathrm{c}}$ as I believe it to be an outlier. Although there are too few data to warrant firm conclusions, both materials appear to have decomposed. Insomuch as anything can be stated on the basis of three points, correspondence of theory and experiment is surprisingly good.

\section{SUMMARY}

I have compared our existing cushion EOS to data, and reviewed some of the elements that went into their construction. I take away several things from this exercise.

- The experimental basis for constructing an inert EOS for these materials is slender. Shock data were all that I could find for SX358 and S5370, meaning that use of anything more relies on their similarity to Sylgard. The comparison is reasonable based on composition, but is somewhat belied by the different property values drawn from the inert tables. I believe the ambient moduli and expansion coefficients as given by 7970 and 7980 should be almost identical, and that Gruneisen gamma in both cases is too small by a factor of at least 2. See section II and Table II for details.

- The compositions used to build 7971 and 7981 are probably as accurate as could be hoped, and any correction would have minor effect. See section III and Tables III-V for details.

- There is very little difference between inert and product Hugoniots as read from 7970/1 and 7980/1. In each case, Hugoniots centered at the reference density match the lowpressure data well. At high pressure, they match well the data for Sylgard. Sylgard products look to be slightly stiffer than those of S5370 (consistent with the inert), but the data are few and relatively scattered. Recent shots by Dattelbaum almost certainly do not reach the threshold for decomposition, meaning there are no products data for full-density SX358. See section IV and Figures 3-8 for details.

\footnotetext{
${ }^{c}$ Number 2s-287
} 
- Recent gas gun experiments on porous SX358 likely do exceed the threshold for decomposition, which probably drops dramatically with initial porosity. ${ }^{25}$ Agreement between the gas gun results and SESAME 7981 is surprisingly good, although there are very few data to go on. See Section IV and Figures 9-10 for details.

- I can think of a few possible lines of improvement. Additional gas gun experiments at pressures exceeding the decomposition threshold would help to nail down the products locus for S5370 and SX358. On the theory side, improvement of the chemical equilibrium solution or the extension procedure might be helpful. Sadly, these are all worthy but obviously non-trivial endeavors.

\section{REFERENCES}

${ }^{1}$ S. P. Lyon and J. D. Johnson, "SESAME: The Los Alamos National Laboratory Equation of State Database," LA-UR-92-3407 (Los Alamos National Laboratory, 1992).

${ }^{2}$ J. Abdallah, "User's Manual for Grizzly," LA-10244-M (Los Alamos National Laboratory, 1984).

${ }^{3}$ G. I. Kerley, "User's Manual for Panda: A Computer Code for Calculating Equations of State," LA-8833-M (Los Alamos National Laboratory, 1981).

${ }^{4}$ Scott Crockett, private communication. (2015)

${ }^{5}$ D. A. Mcquarrie, Statistical Mechanics (University Science Books, 1976) . See Sec. 11-3.

${ }^{6}$ R. P. Feynman, N. Metropolis, and E. Teller, Phys. Rev. 75 (1949).

${ }^{7}$ R. D. Cowan and J. Ashkin, Phys. Rev. 105 (1957).

${ }^{8}$ B. Wunderlich and H. Baur, Adv. Polym. Sci. 7, 151 (1970).

${ }^{9}$ D. M. Dattelbaum, J. D. Jensen, A. M. Schwendt, E. M. Kober, M. W. Lewis, and R. Menikoff, J. Chem. Phys. 122, 144903 (2005).

${ }^{10}$ Chosen to be consistent with measured values of $\alpha, C_{p}$, and the extrapolation to zero particle velocity of the Hugoniot data.

${ }^{11}$ F. Dowell, "A Simple EOS for the Silicone Rubber Sylgard 184," LA-10164-MS (Los Alamos National Laboratory, 1984).

${ }^{12}$ F. W. DuBois, "Properties of materials table," internal memorandum, Los Alamos National Laboratory (1984). 
${ }^{13}$ Dow-Corning, "Information About Electrical/Electronic Materials: Sylgard 182, 183, 184, and 185 Resins for Potting and Encapsulating," Technical Bulletin 61-283 (Dow-Corning, 1974), as cited in 11.

${ }^{14}$ D. M. Dattelbaum, D. Robbins, S. Sheffield, and R. Alcon, "Hugoniot Measurements of $1.1 \mathrm{~g} / \mathrm{cm}^{3}$ SX358," (2006), los Alamos National Laboratory.

${ }^{15}$ J. S. Rowlinson and F. L. Swinton, Liquids and Liquid Mixtures, 3rd ed. (Butterworth Scientific, 1982) . A macroscopic treatment of ideal mixtures is given in 4.3, a microscopic one in 8.2 .

${ }^{16}$ S. Crockett, "Extending High Explosive Equations of State," LA-UR-06-5738 (Los Alamos National Laboratory, 2006).

${ }^{17}$ M. Ross, J. Chem. Phys. 71, 1567 (1979).

${ }^{18}$ T. Stephens, "Aging Mechanisms in RTV Silicone Foams," LA-UR-06-0740 (Los Alamos National Laboratory, 2006).

${ }^{19}$ A. J. Barry, J. Appl. Phys. 17, 1020 (1946).

${ }^{20}$ Dow Corning, private communication. (2015)

${ }^{21}$ S. P. Marsh, ed., LASL Hugoniot Data (University of California Press, 1980).

${ }^{22}$ C. E. Morris, J. N. Fritz, and R. G. McQueen, J. Chem. Phys. 80, 5203 (1984).

${ }^{23}$ C. E. Morris, E. D. Loughran, G. F. Mortensen, and M. S. Shaw, in Shock Compression of Condensed Matter - 1989 (1990) pp. 687-690.

${ }^{24}$ W. J. Carter and S. P. Marsh, "Hugoniot Equation of State of Polymers," LA-13006-MS (Los Alamos National Laboratory, 1995).

${ }^{25}$ D. M. Dattelbaum, J. D. Coe, C. B. Kiyanda, R. L. Gustavsen, and B. M. Patterson, J. Appl. Phys. 115, 174908 (2014).

${ }^{26} \mathrm{R}$. Menikoff, in Shock Wave Science and Technology Reference Library, edited by Y. Horie (Springer, 2007) Chap. 4.

${ }^{27}$ D. M. Dattelbaum, J. D. Coe, P. A. Rigg, R. J. Scharff, and J. T. Gammel, J. Appl. Phys. 16, 194308 (2014).

${ }^{28} \mathrm{I}$ don't know of a standard reference for the code, although much of the underlying physics is covered in Refs. 1-3. The best source for the software is its online documentation, available at https ://pitchfork.lanl.gov/wiki/index.php/Main_Page.

${ }^{29}$ Sam Shaw, unpublished work. 


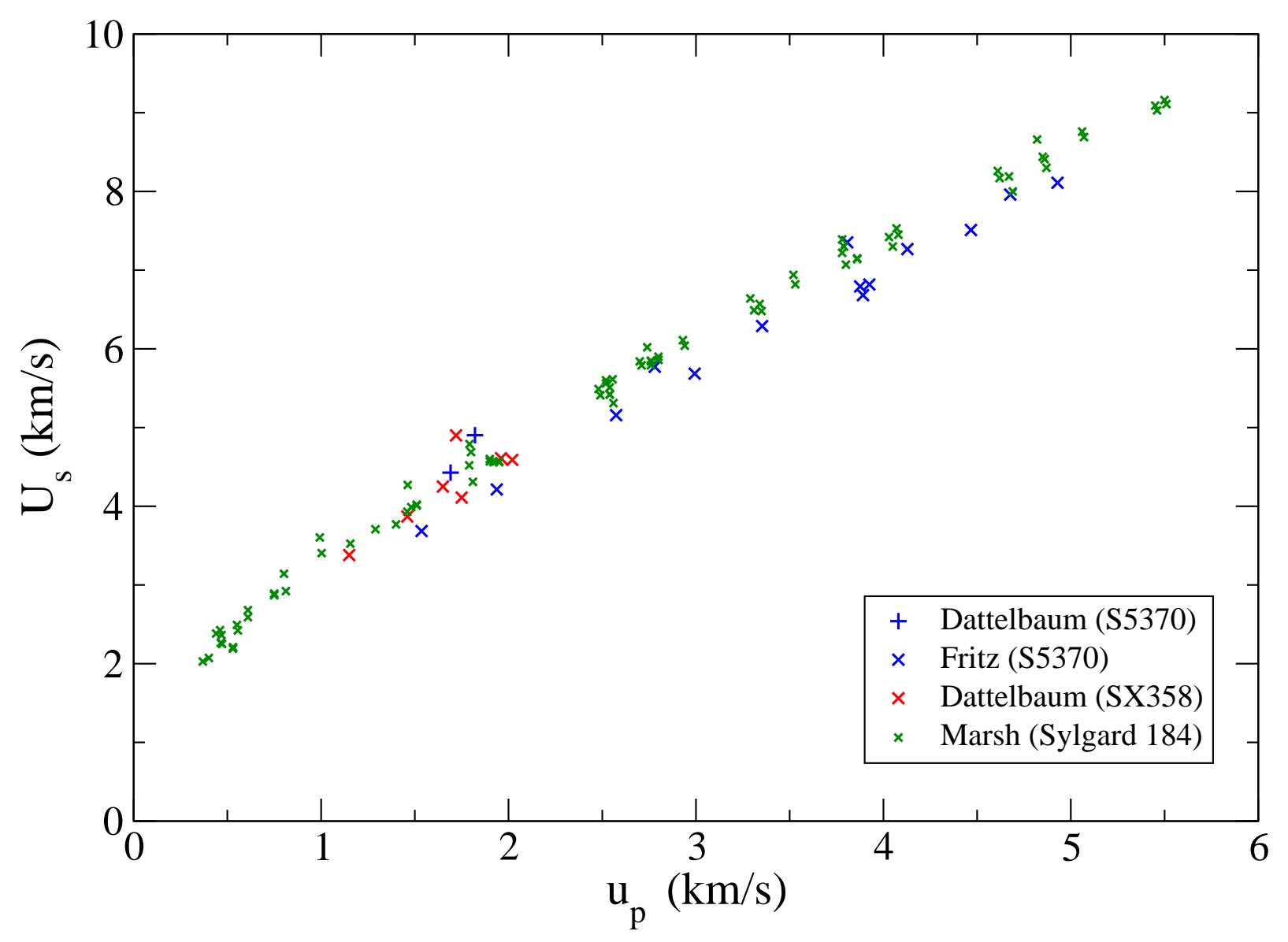

FIG. 1. Shock data in the $U_{s}-u_{p}$ plane for stress cushion and related materials. 


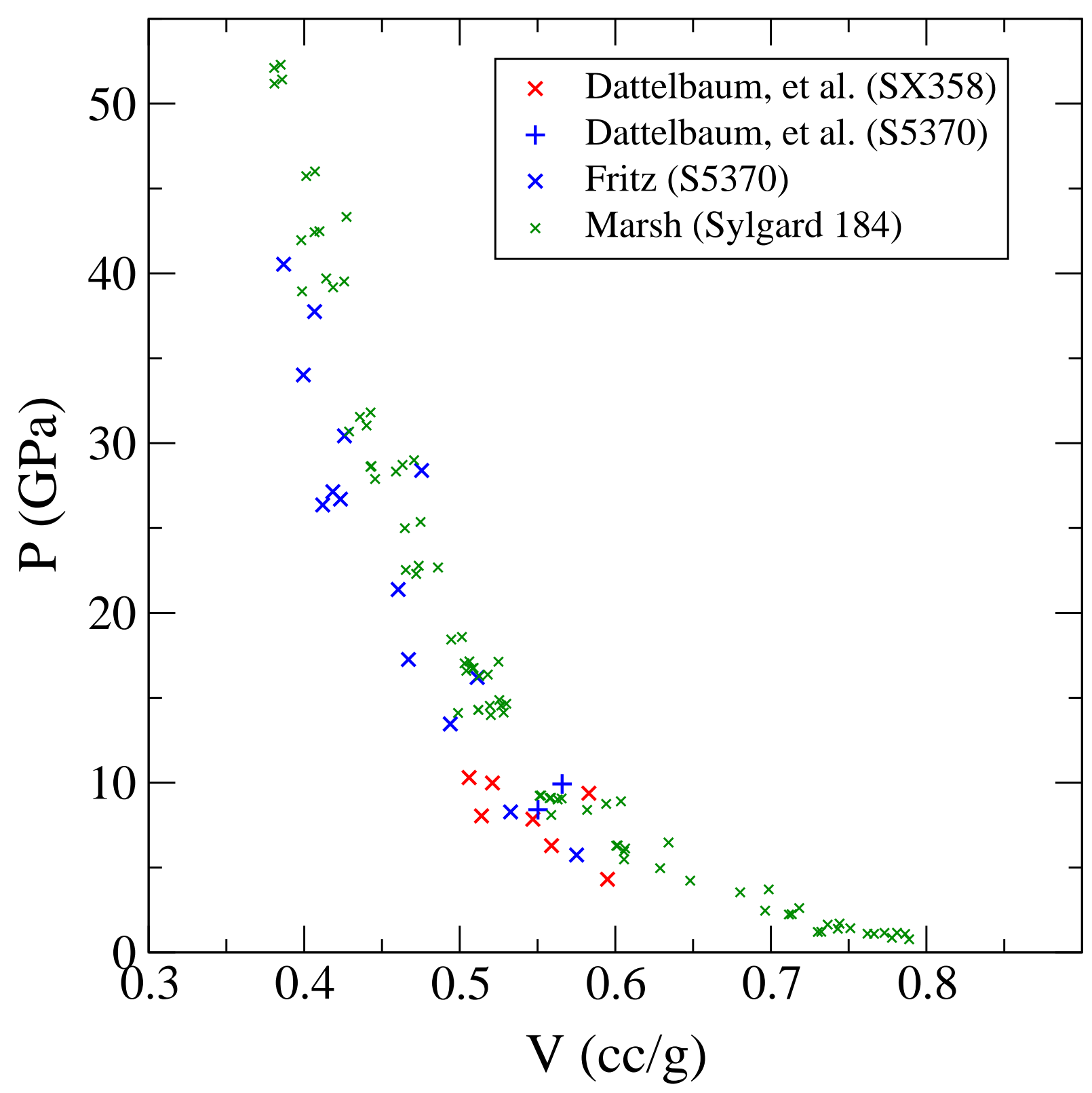

FIG. 2. Shock data in the $P-V$ plane for stress cushion and related materials. 


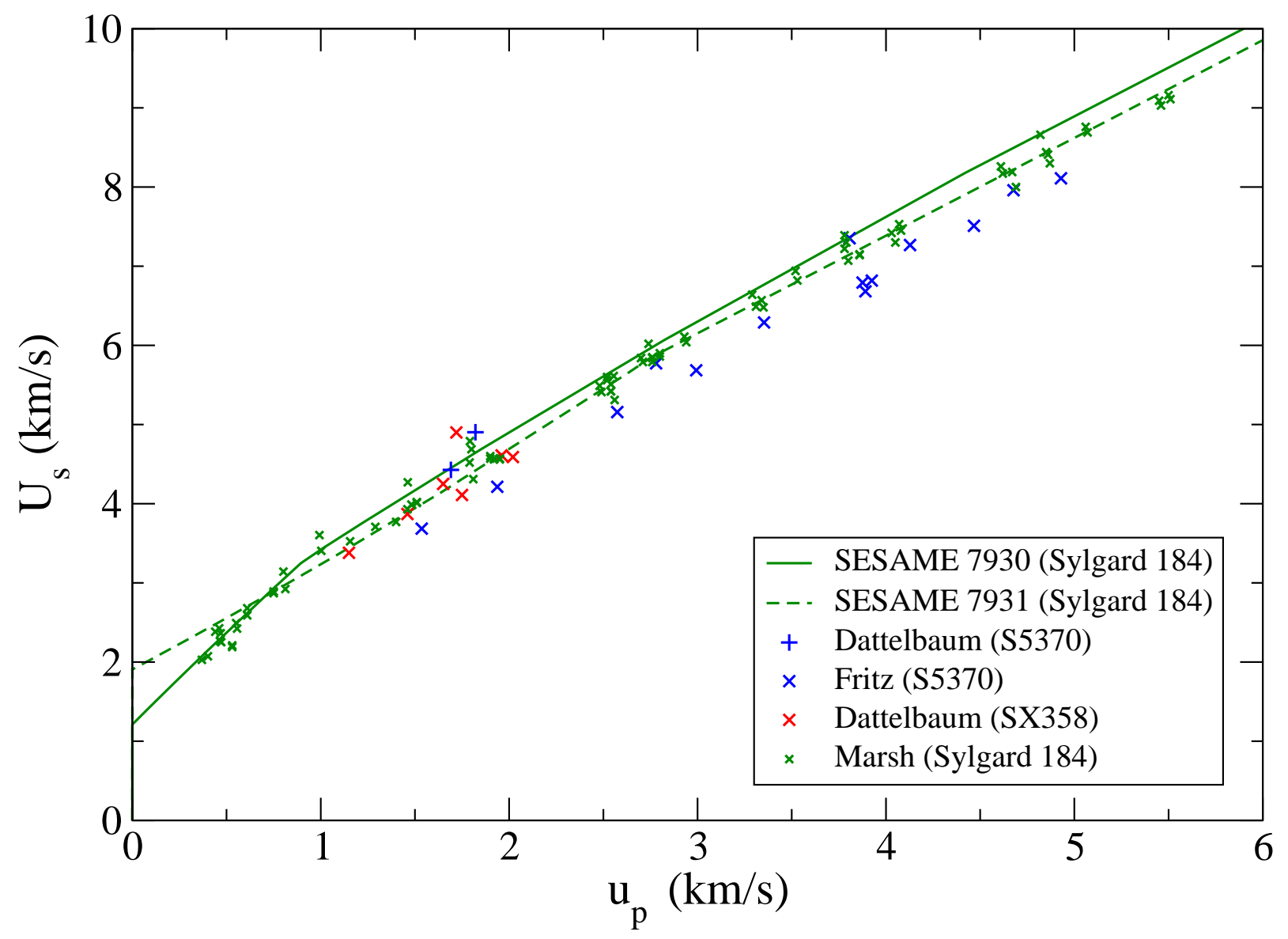

FIG. 3. Shock data in the $U_{s}-u_{p}$ plane for stress cushion and related materials, as compared with the Hugoniots predicted by SESAMEs 7930 and 7931 for Sylgard 184 . 


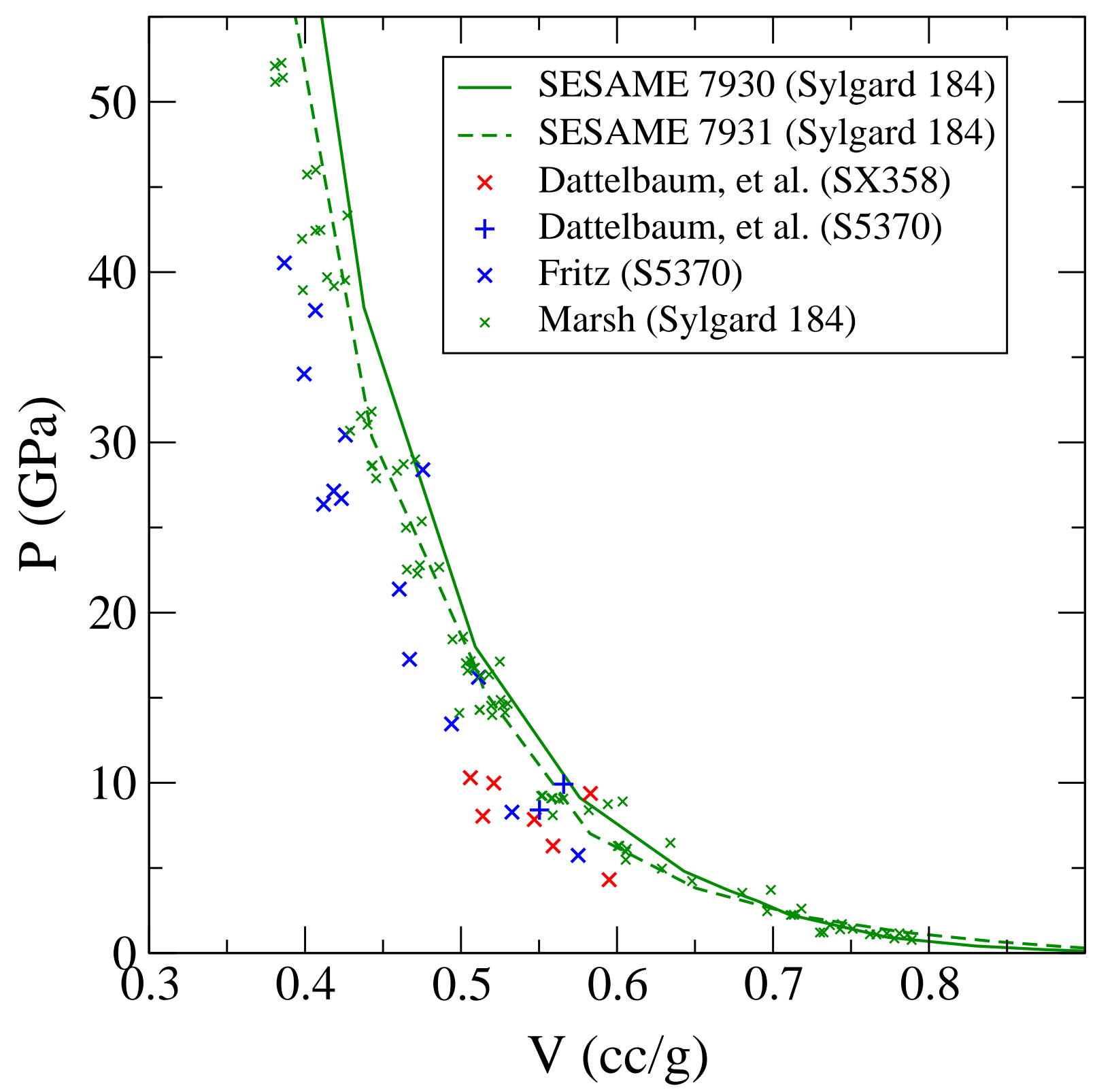

FIG. 4. Shock data in the $P-V$ plane for stress cushion and related materials, as compared with the Hugoniots predicted by SESAMEs 7930 and 7931 for Sylgard 184 . 


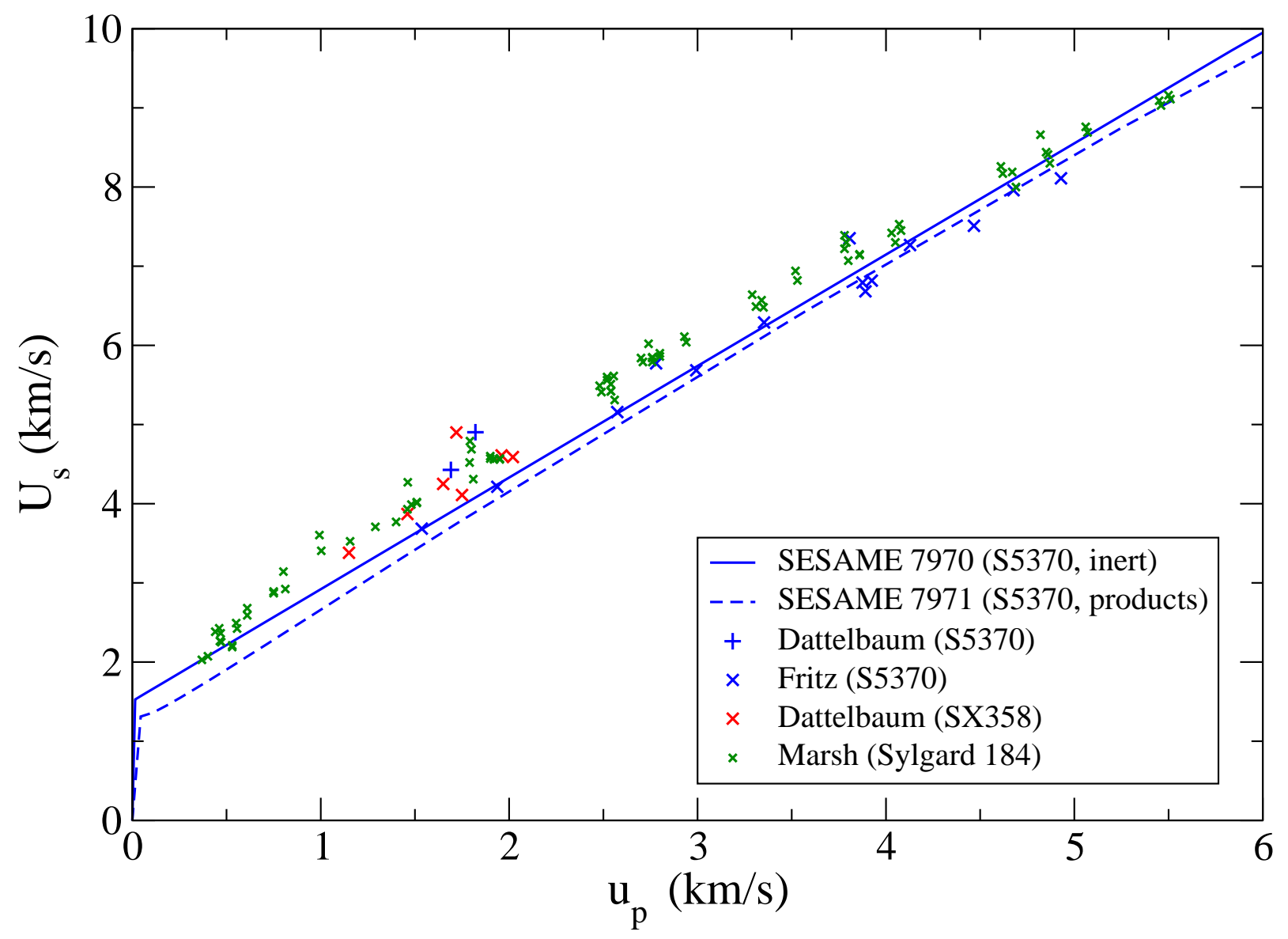

FIG. 5. Shock data in the $U_{s}-u_{p}$ plane for stress cushion and related materials, as compared with the Hugoniots predicted by SESAMEs 7970 and 7971 for S5370. 


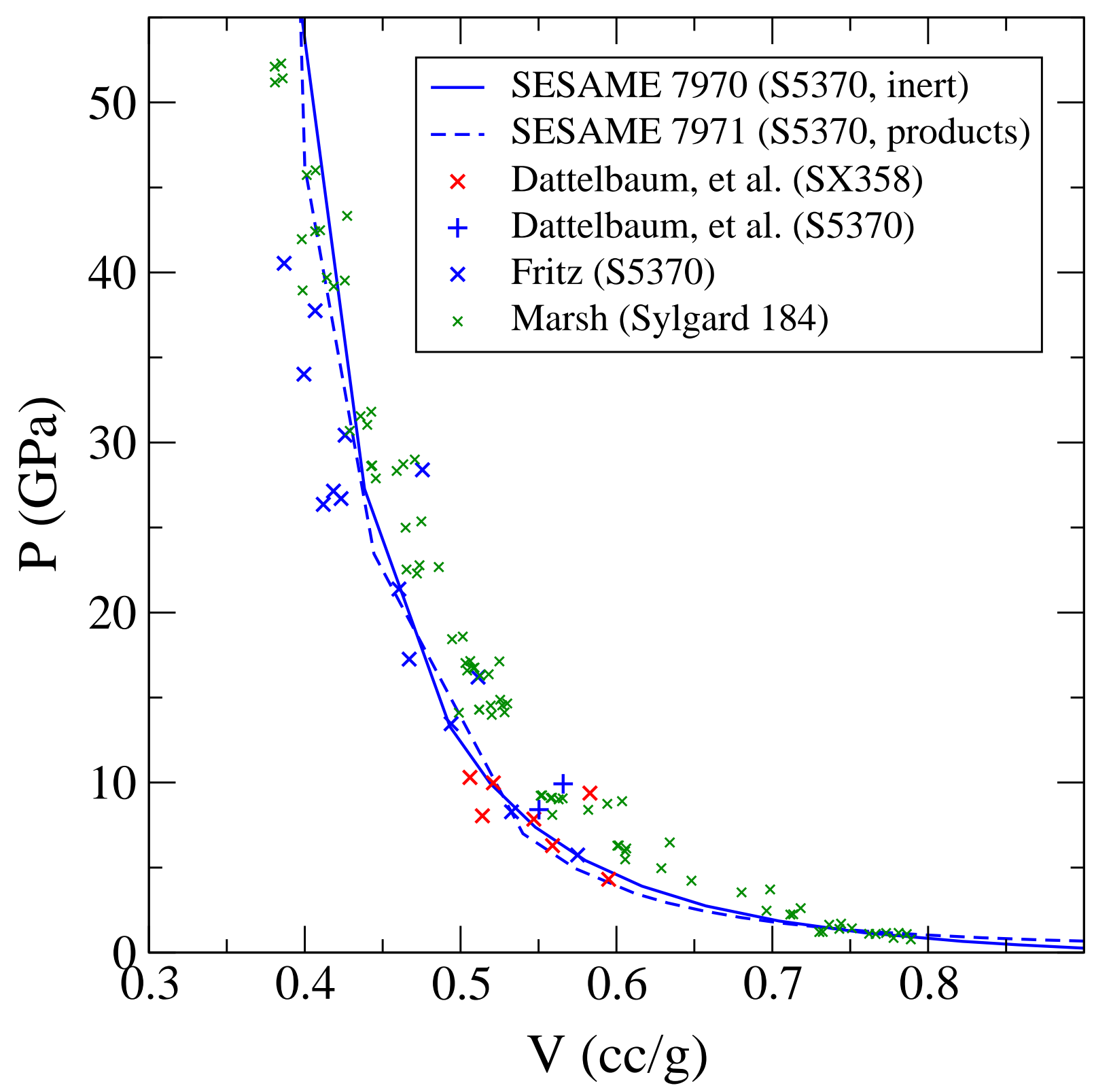

FIG. 6. Shock data in the $P-V$ plane for stress cushion and related materials, as compared with the Hugoniots predicted by SESAMEs 7970 and 7971 for S5370. 


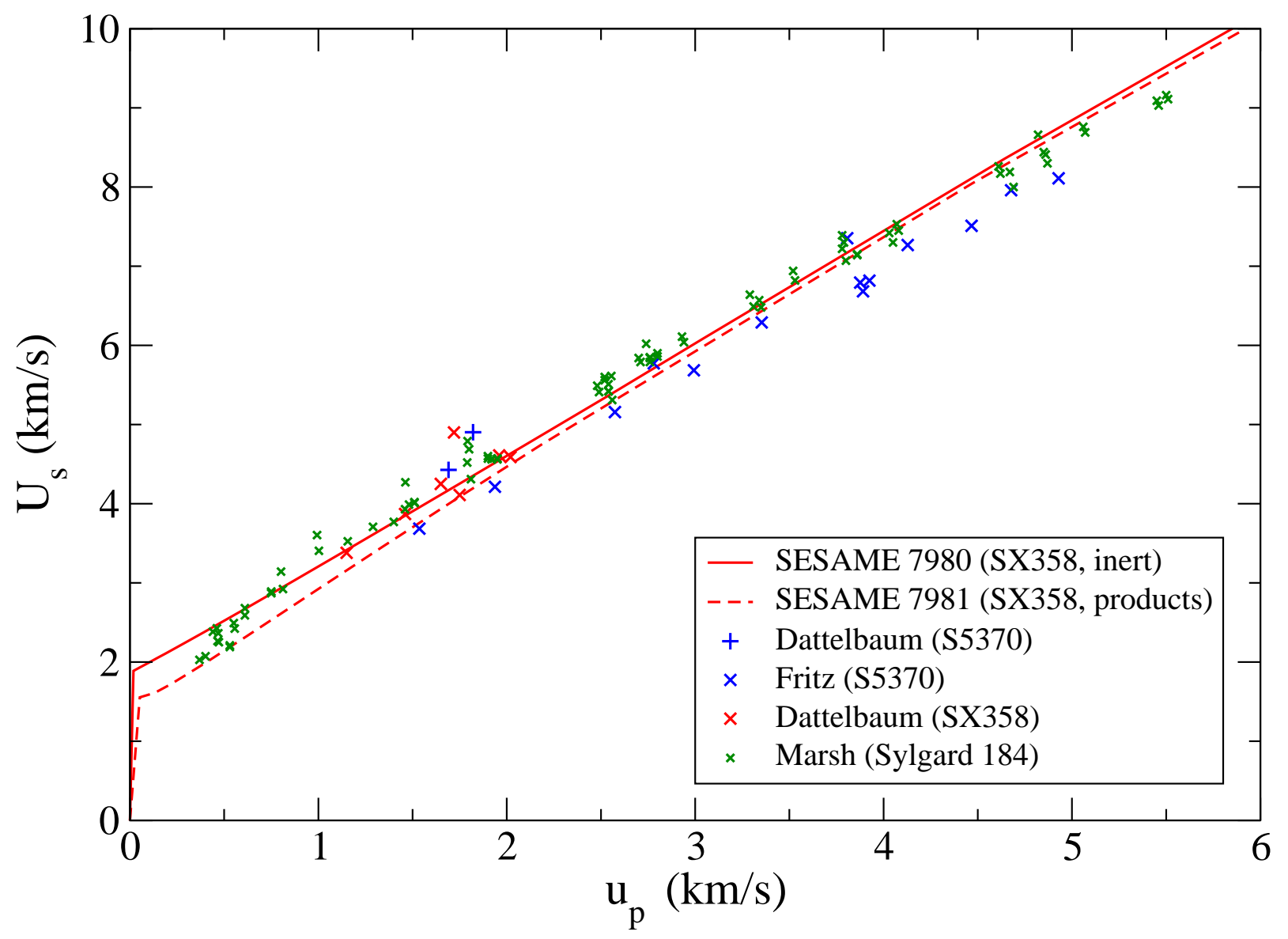

FIG. 7. Shock data in the $U_{s}-u_{p}$ plane for stress cushion and related materials, as compared with the Hugoniots predicted by SESAMEs 7980 and 7981 for SX358. 


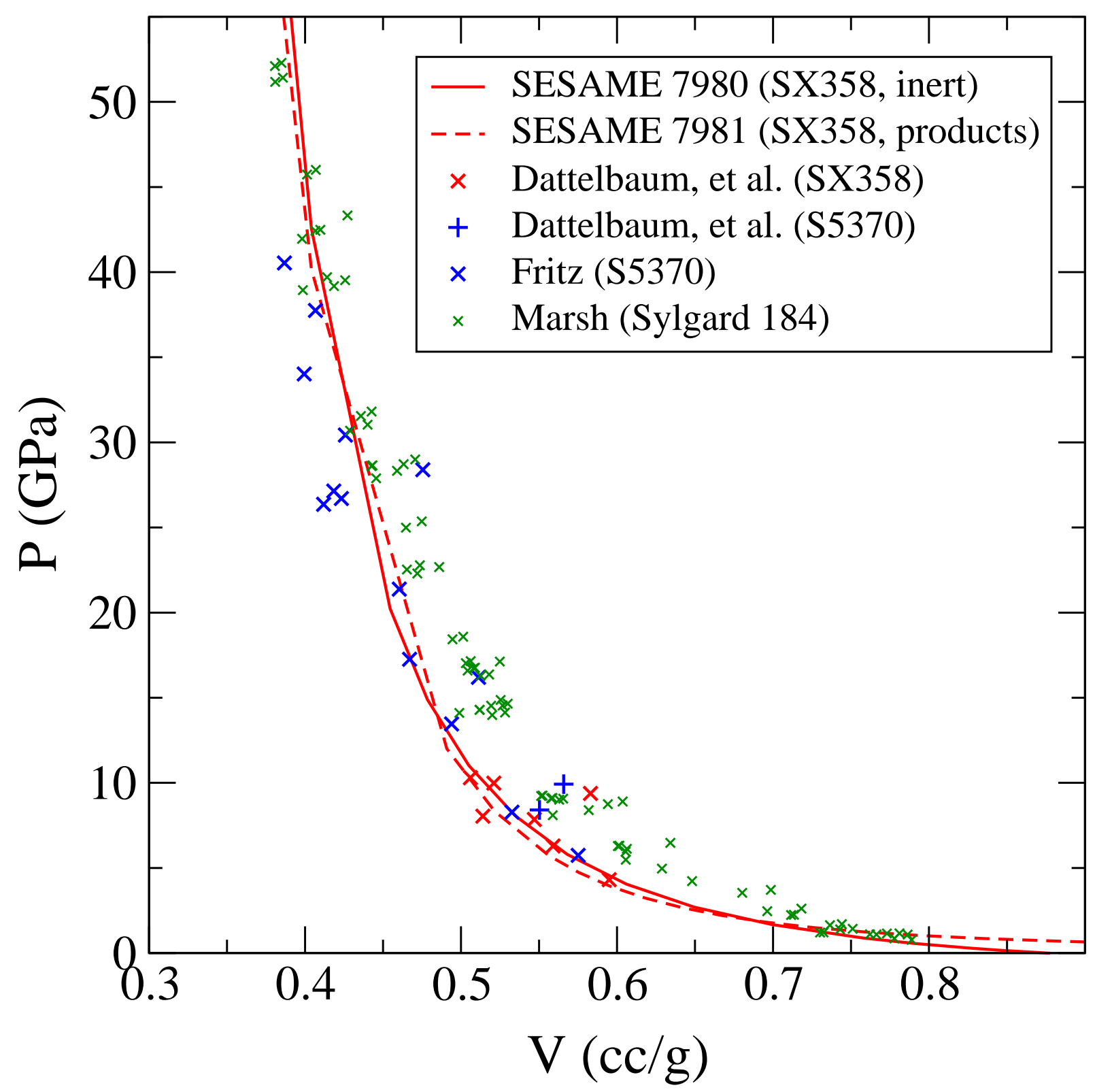

FIG. 8. Shock data in the $P-V$ for stress cushion and related materials, as compared with the Hugoniots predicted by SESAMEs 7980 and 7981 for SX358. 


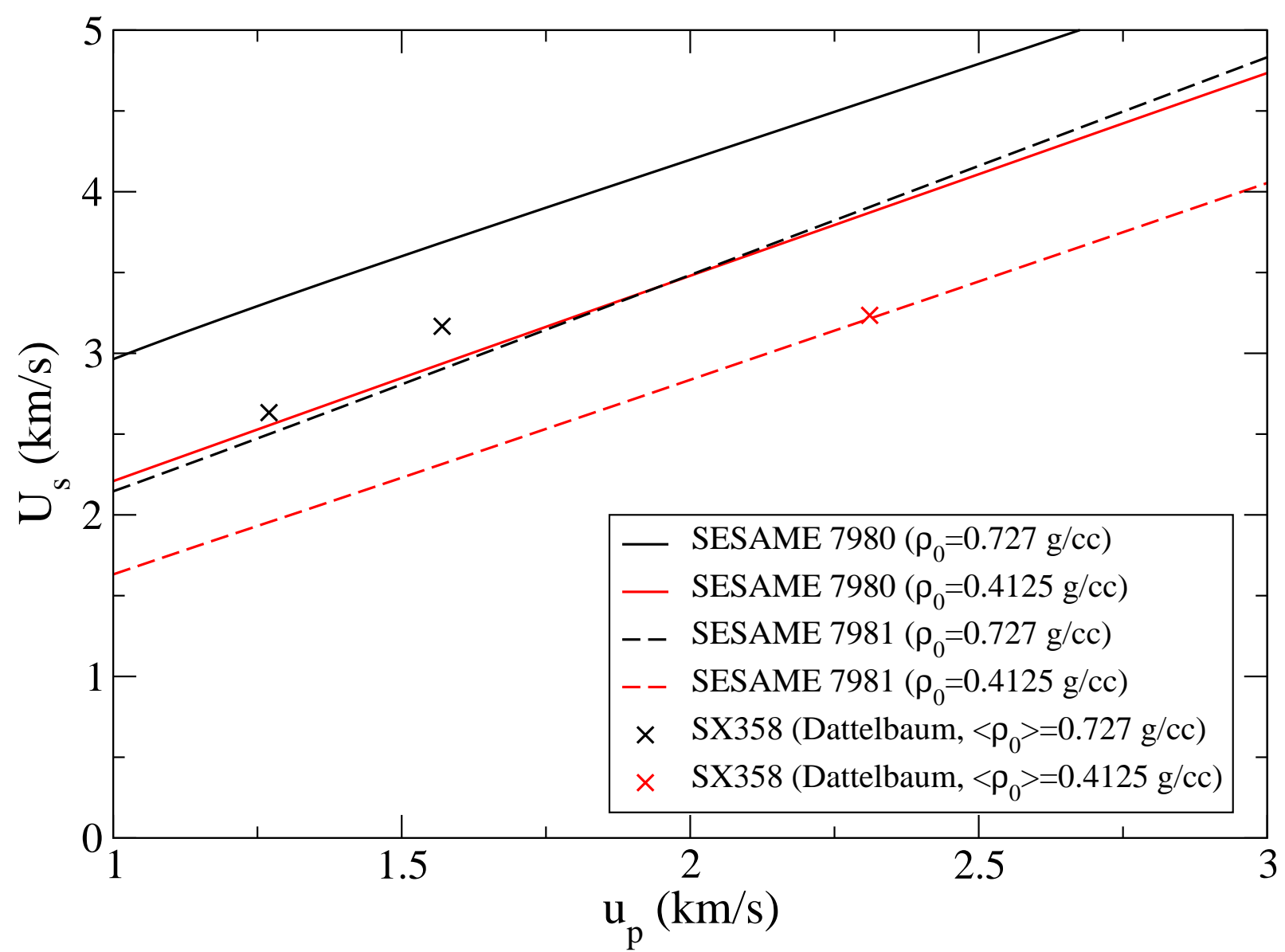

FIG. 9. Shock data in the $U_{s}-u_{p}$ plane for porous SX358, as compared with the Hugoniots predicted by SESAMEs 7980 and 7981 . 


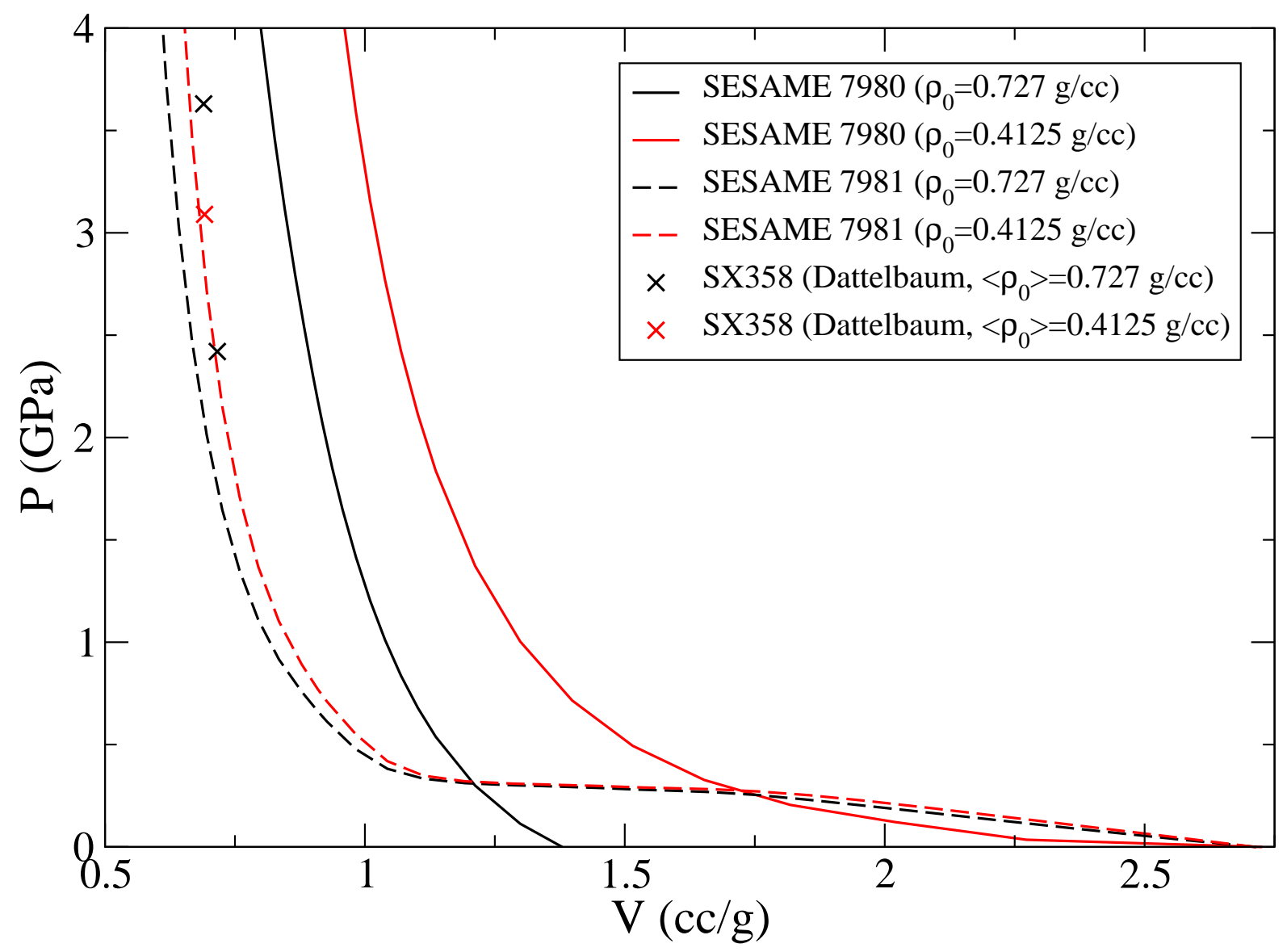

FIG. 10. Shock data in the $P-V$ plane for porous SX358, as compared with the Hugoniots predicted by SESAMEs 7980 and 7981. 